\title{
Funções Executivas na Infância: Avaliação e Dados Normativos Preliminares para Crianças Portuguesas em Idade Pré-escolar
}

\author{
Executive Functions in Childhood: Assessment and Preliminary Normative Data for \\ Portuguese Preschoolers
}

\author{
Ana Paula Prust Pereira ${ }^{1}$, Natália Martins Dias $^{2}$, Alexandra M. Araújo ${ }^{3}$ e Alessandra Gotuzo \\ Seabra $^{4}$
}

\begin{abstract}
Resumo
O presente estudo investigou evidências de validade do Teste de Atenção por Cancelamento (TAC) e Teste de Trilhas para pré-escolares (TT-PE) e disponibilizou normas preliminares para pré-escolares portugueses. Participaram 105 crianças, com 5 e 6 anos, avaliadas no TAC (atenção) e TT-PE (flexibilidade cognitiva). Houve efeito da idade para o TAC, com melhor desempenho das crianças de 6 anos, sugerindo trajetórias de desenvolvimento distintas para as habilidades avaliadas. Relações baixas a altas foram observadas entre índices de um mesmo instrumento, e baixas a moderadas entre desempenhos nos dois testes. Houve diferença significativa entre desempenhos da amostra portuguesa e a normatização brasileira na maioria das medidas. Pontuações-padrão foram calculadas. São fornecidas evidências de validade e normalização preliminar para instrumentos de avaliação de crianças portuguesas em idade pré-escolar.
\end{abstract}

Palavras-chave: neuropsicologia, educação infantil, pré-escolar, padronização do teste, funções executivas

\begin{abstract}
The present study investigated validity evidence of the Cancellation Attention Test (CAT) and Trail Making Test for Preschoolers (TMT-P) and provided preliminary standards for Portuguese preschoolers. A total of 105 five and six year-old children were evaluated with the CAT (attention) and the TMT-P (cognitive flexibility). There was an age effect for TAC, with better performance of 6-years-old, suggesting different developmental trajectories for the assessed skills. Low to high relationships were observed between indices of the same instrument, and low to moderate between performances in both tests. There was a significant difference between the Portuguese sample performance and the Brazilian standards in most of the measures. Standard scores were calculated. Validity evidence and preliminary standards for assessment instruments in Portuguese preschoolers are provided.
\end{abstract}

Keywords: neuropsychology, early childhood education, preschool children, test standardization, executive functions

Agradecimentos: Capes (APPP); CNPq (NMD; AGS)

${ }^{1}$ Pedagoga, Doutora em Distúrbios do Desenvolvimento pela Universidade Presbiteriana Mackenzie. Oficial Militar Psicopedagoga da Divisão de Ensino. Academia da Força Aérea Brasileira - AFA, Pirassununga, Brasil. Estrada de Aguaí, s/n $\mathrm{n}^{\circ}$ - Campo Fontenelle CEP 13643-000 Pirassununga-SP, Brasil. Tel.: +55193565-7487. Email: anaprustpsicopedagoga @gmail.com

${ }^{2}$ Psicóloga, Doutora e Pós-Doutora em Distúrbios do Desenvolvimento pela Universidade Presbiteriana Mackenzie. Docente do Departamento de Psicologia. Universidade Federal de Santa Catarina - UFSC, Florianópolis, Brasil. Bolsista de produtividade em pesquisa do CNPq. E-mail: natalia_mdias@yahoo.com.br

${ }^{3}$ Doutora em Psicologia, Professora Auxiliar. Departamento de Psicologia e Educação, Universidade Portucalense, Porto, Portugal. E-mail: amaraujo@upt.pt

${ }^{4}$ Psicóloga, Docente do Programa de Pós-graduação em Distúrbios do Desenvolvimento. Universidade Presbiteriana Mackenzie, São Paulo, Brasil. Bolsista de produtividade CNPq. E-mail: alessandragseabra@gmail.com 


\section{Introdução}

As funções executivas (FE) referem-se a um conjunto de processos mentais responsáveis pela capacidade de autogestão do indivíduo, possibilitando o controlo e a regulação do comportamento, da emoção e da cognição (Barros \& Hazin, 2013; Diamond, 2013, 2014). Há relativo consenso na literatura de que as FE não são uma habilidade unitária, integrando diferentes componentes. Isso tem sido verificado desde o estudo pioneiro de Miyake, Friedman, Emerson, Witzki, Howerter e Wager (2000) que, numa amostra de adultos, identificou três habilidades interdependentes: atualização (updating), inibição e alternância (shifting). Estudos subsequentes têm corroborado esses achados, sobretudo em adolescentes e adultos (Friedman \& Miyake, 2017). No entanto, esta estrutura fatorial parece ser influenciada por aspectos do desenvolvimento. Estudos sugerem que as $\mathrm{FE}$ são menos diferenciadas na infância, havendo maior suporte para uma estrutura unifatorial, sobretudo no período pré-escolar. Com o desenvolvimento, as distintas habilidades passam a tornar-se mais interdependentes, apesar de ainda não haver consenso sobre a faixa etária exata em que essa diferenciação ocorre (Brydges, Fox, Reid, \& Anderson, 2014; Garon, Bryson, \& Smith, 2008; Wiebe et al., 2011).

Com base no modelo de Miyake et al. (2000), Diamond (2013) sugeriu uma estrutura conceitual, bastante aceite atualmente, que sugere que as FE são compostas por três habilidades básicas: inibição, memória de trabalho e flexibilidade cognitiva. Este estudo terá como foco duas destas habilidades: a inibição, que se refere à capacidade de inibir comportamentos inadequados, bem como a atenção a distratores, incluindo, portanto, a atenção seletiva; e a flexibilidade cognitiva, ou seja, a habilidade de tomar diferentes perspectivas e mudar o foco atencional entre tarefas (Diamond, 2013).

Tais habilidades são necessárias para controlar emoções e impulsos, para prestar atenção e para a adaptação a uma tarefa ou ao ambiente físico, social e educacional (Engel de Abreu et al., 2015), apresentando-se como componentes importantes para o sucesso escolar. De facto, há evidências de que as FE são relevantes para facilitar a aprendizagem de diversos conteúdos escolares, bem como para o julgamento e comportamento social (Campbell, Pungello, Miller-Johnson, Burchinal, \& Ramey, 2001; Heckman, 2006; Diamond, 2013; Nesbitt, Farran, \& Fuhs, 2015; Seabra et al., 2014).

As FE iniciam o seu desenvolvimento muito cedo no ciclo vital. A inibição (e especificamente a atenção seletiva) parece ser uma das primeiras habilidades a emergir, já por volta de 6 meses de idade. Ao final do primeiro ano, por exemplo, a criança começa a ser capaz de manter o foco de atenção em uma tarefa, apesar de algumas distrações. Por volta dos 4 ou 5 anos, torna-se mais hábil em inibir os seus comportamentos e aos 7 anos são eficazes em lidar com distratores e focalizar a atenção. Um período importante para o desenvolvimento da inibição parece dar-se entre os 3 e 5 anos, continuando até à adolescência (Best \& Miller, 2010; Center on the Developing Child at Harvard University [CDCHU], 2011; Dias \& Seabra, 2013). Por seu lado, a flexibilidade parece desenvolver-se um pouco mais tarde, iniciando-se por volta do final do primeiro ano de vida. Por volta dos 2 a 5 anos, as crianças começam a adequar $o$ seu comportamento a diferentes regras ou ambientes, apesar desta habilidade continuar a desenvolver-se de forma intensa até próximo dos 12 anos, progredindo ainda até aproximadamente aos 18 anos. Alguns resultados da investigação sugerem que a idade entre os 5 e 7 anos parece ser um período importante, com uma maior progressão da flexibilidade, apesar do desenvolvimento contínuo ao longo dos anos vindouros (Best \& Miller, 2010; CDCHU, 2011; Dias \& Seabra, 2013).

A maioria dos autores tende a concordar que o período pré-escolar parece ser um período relevante e de rápido desenvolvimento das FE (Barros \& Hazin, 2013; Best \& Miller, 2010; Bierman \& Torres, 2016; CDCHU, 2011; Diamond, 2014), havendo inclusive ênfase crescente na literatura internacional para a promoção destas habilidades nesta faixa etária (Blair, 2016; Diamond \& Lee, 2011; Dias \& Seabra, 2015). Assim, o período pré-escolar parece ser um período oportuno para a avaliação e identificação precoce de atrasos, tanto quanto de estimulação para o desenvolvimento de tais habilidades como em implicações para adaptação 
escolar posterior.

Neste sentido, a avaliação neuropsicológica infantil, área na qual se insere este estudo, pode trazer uma contribuição importante para a avaliação precoce, permitindo a identificação de crianças em risco de problemas no seu desenvolvimento. Por exemplo, sabe-se que as FE estão comprometidas em diversos quadros dos Transtornos do Neurodesenvolvimento. Já em idade pré-escolar, défices nas FE são observadas no Transtorno do Espectro do Autismo - TEA e podem predizer a habilidade da Teoria da Mente, relevante para o desenvolvimento de interações sociais adaptativas (Kimhi, Shoam-Kugelmas, Ben-Artzi, Ben-Moshe, \& Bauminger-Zviely, 2014). Mais ainda, estes associam-se ao risco para o Transtorno de Défice de Atenção e Hiperatividade - TDAH (Pauli-Pott \& Becker, 2011).

Evidências também apontam que, no período pré-escolar, as FE estão associadas a índices de comportamento e saúde mental infantil (Dias, Trevisan, León, Prust, \& Seabra, 2017) e podem predizer sintomas de ansiedade e depressão no $4^{\circ}$ ano do Ensino Fundamental, ou seja, são fatores de risco para psicopatologia infantil (Nelson, Kidwell, Nelson, Tomaso, Hankey, \& Espy, 2018). Ao lado disso, a literatura tem documentado evidências da contribuição das FE para a aprendizagem escolar, sobretudo medidas em termos de desempenho em leitura e matemática (Nesbitt et al., 2015; Seabra et al., 2014).

Assim, a avaliação dessas habilidades na faixa etária pré-escolar pode auxiliar no delineamento de um perfil de dificuldades e orientação da intervenção, seja no âmbito clínico quando as dificuldades já estiverem presentes (reabilitação), mas sobretudo no domínio escolar, numa perspectiva de estimulação e prevenção de problemas (de aprendizagem e/ou de comportamento) futuros.

No entanto, avaliar essas habilidades nesta faixa etária muitas vezes pode ser um desafio. Numa revisão sobre o perfil das pesquisas sobre avaliação de FE na infância, Barros e Hazin (2013) identificaram apenas nove estudos, entre um total de 25 selecionados na revisão, conduzidos com crianças em idade pré-escolar. Os autores destacaram alguns pontos, nomeadamente:
1) o uso de diferentes medidas de avaliação, o que impossibilita a comparação entre estudos e dificulta a apresentação de resultados na direção de dados normativos para o desenvolvimento típico das $\mathrm{FE}$; e 2) a pequena quantidade de artigos identificados. Além disso, uma análise dos estudos levantados pelos referidos autores revela, àquela data, a inexistência de estudos portugueses ou com amostras de crianças portuguesas entre os artigos selecionados na revisão. Para os autores, os seus resultados refletem a carência de tarefas padronizadas para avaliar as FE na primeira e segunda infância.

No contexto português, vários estudos têm sido conduzidos com foco na avaliação das FE na infância. Um destes estudos realizou a tradução e adaptação do Children's Color Trails Test (Llorente, Williams, Satz, \& D'Elia, 2003), que envolve atenção e flexibilidade, para a avaliação de crianças com idades entre os 6 e 11 anos. $\mathrm{O}$ mesmo estudo também procurou delinear o perfil de funcionamento executivo de crianças entre os 6 e 10 anos a partir de uma bateria de instrumentos, tendo observado uma progressão no desempenho em todos os instrumentos em função da escolaridade e um efeito inconsistente do sexo, evidenciado apenas numa tarefa de memória (Pinto, 2008). Outro estudo realizou a tradução e adaptação do Behavior Rating Inventory of Executive Functions (BRIEF), aplicando-o a uma amostra de crianças de 10 a 13 anos (Ribeiro, Cruz-Santos, \& Miranda-Correia, 2012). Por seu lado, as tarefas de fluência verbal semântica e fonémica também têm sido utilizadas, como no estudo de Moura, Simões e Pereira (2013), que avaliaram crianças com idades de 7 a 10 anos, observando um desempenho crescente em função da idade das crianças, apesar de não encontrarem um efeito significativo do sexo.

Apesar dos resultados destes estudos prévios, verifica-se que a faixa etária pré-escolar é ainda pouco abordada. Uma exceção a esta tendência é o estudo de Teixeira (2012), que avaliou crianças de 3 e 4 anos no The Shape School (Espy, Bull, Martin, \& Stroup, 2006), um instrumento que avalia inibição e flexibilidade. Neste estudo, não se verificou um efeito do sexo e os resultados sugeriam que a habilidade de flexibilidade parece ser preditora da compreensão de frases complexas e nomeação de imagens. Ainda no contexto etário 
pré-escolar, as autoras Carapito, Ribeiro e Pereira (2015) ressaltam a necessidade de estudos nesta etapa do desenvolvimento, sendo que no estudo analisaram o impacto do envolvimento parental na escola nos resultados académicos através da versão para pais do Questionário de Envolvimento Parental no Jardim-de-Infância - QEPJI (Carrapito et al., 2015). Face à inexistência de questionários de avaliação do envolvimento parental na escola para esta etapa da escolaridade, o estudo teve por objetivo apresentar alguns resultados psicométricos da versão para pais do QEPJI. Os resultados obtidos sugerem que este é um instrumento adequado para a avaliação do envolvimento parental em contexto pré-escolar. As autoras verificaram que o entusiasmo parental e um estilo educativo positivo revelam ser os melhores preditores dos resultados académicos.

Com o objetivo de apresentar o processo de tradução e adaptação do Metacognitive Awareness Inventory (MAI) para a população portuguesa e apresentar os resultados relativos ao estudo das qualidades psicométricas da versão adaptada, Bártolo-Ribeiro, Simões e Almeida (2016) aplicaram o instrumento a participantes com número igual ou superior a 10 anos de escolaridade. O MAI (Schraw \& Dennison, 1994) foi originalmente desenvolvido por Schraw e Dennison (1994) para ser aplicado a adolescentes e adultos, com o objetivo de identificar o nível de consciência cognitiva de quem se encontra num processo de aprendizagem. A fidedignidade da versão portuguesa do inventário global foi elevada; contudo, os valores de consistência interna para algumas das subescalas tomadas isoladamente foram inferiores, porém dentro do recomendável para este tipo de instrumentos (Bártolo-Ribeiro et al., 2016).

Recentemente, assistiu-se à publicação da Bateria de Avaliação Neuropsicológica de Coimbra (BANC, Simões et al., 2016), composta por 15 testes neuropsicológicos e com normas para crianças e adolescentes de 5 a 15 anos portugueses. A Bateria, entre outras áreas, avalia atenção e FE. Especificamente, os instrumentos para avaliação da atenção e FE incluem tarefas de Fluência Verbal, Torre, Cancelamento de Sinais e Trilhas. $\mathrm{O}$ instrumento mostrou bons índices de fidedignidade (teste-reteste) e evidências de validade por relação com outras variáveis e por adequação de sua estrutura interna (Simões et al., 2016). Em um dos estudos com o instrumento, com amostra entre os 7 e 15 anos, os autores relataram um bom ajuste do modelo de três fatores correlacionados (memória, linguagem, atenção e FE), corroborando as evidências de validade de construto da BANC, que se tem mostrado adequada para o estudo do desenvolvimento neuropsicológico típico e atípico na infância e adolescência (Moura et al., 2017).

No contexto brasileiro, também algumas medidas têm sido desenvolvidas e adaptadas com foco na avaliação de crianças em idade préescolar, entre elas o Teste de Atenção por Cancelamento (Montiel \& Seabra, 2012) e o Teste de Trilhas para pré-escolares (Trevisan \& Seabra, 2012), ambos com propriedades psicométricas satisfatórias verificadas em diversos estudos (sumariados em Seabra \& Dias, 2012). Apesar da existência no contexto português de medidas de FE para crianças (e.g., BANC), seria pertinente dispor de alternativas ao alcance dos profissionais, nomeadamente para a avaliação repetida durante o acompanhamento da intervenção. Mais ainda, dispor das mesmas medidas padronizadas para uso em ambos os países facilitaria comparações e estudos transculturais acerca do desenvolvimento das FE. Contudo, utilizar essas tarefas no contexto português requer adaptações e estudos, uma vez que as FE são habilidades susceptíveis à influência ambiental. Por exemplo, há evidências de que variáveis como o nível socioeconómico, ambiente familiar e estilo de interação entre a criança e os pais (Bernier, Carlson, Deschênes, \& Matte-Gagné, 2012; Engel de Abreu et al., 2015; Hammond, Müller, Carpendale, Bibok, \& Liebermann-Finestone, 2012; Noble et al., Sowell, 2015) podem ter um impacto importante sobre o desenvolvimento das FE. Para além destas variáveis, o contexto cultural também parece ter um papel importante (Carlson, 2009) neste desenvolvimento.

Apesar de o Brasil e Portugal compartilharem o mesmo idioma, existem diferenças relativas a aspectos como a cultura, a qualidade educacional e o nível socioeconómico, que podem impossibilitar o uso direto de medidas brasileiras e das suas normas de desempenho, no contexto português. Neste panorama, após a adequação da linguagem para o português europeu, os objetivos 
deste estudo incluíram: por um lado, investigar evidências de validade por relação com outras variáveis (por mudança no desenvolvimento e relação entre as medidas) para dois instrumentos para a avaliação da atenção, Teste de Atenção por Cancelamento (Montiel \& Seabra, 2012) e flexibilidade cognitiva, Teste de Trilhas para crianças em idade pré-escolar (Trevisan \& Seabra, 2012), numa amostra de crianças portuguesas em idade pré-escolar; e, por outro, disponibilizar dados normativos preliminares para crianças portuguesas em idade pré-escolar.

\section{Método}

\section{Participantes}

Inicialmente, 110 crianças em idade pré-escolar, com idades entre os 5 e 6 anos, foram autorizadas pelos seus encarregados de educação a participar na pesquisa. Todas as crianças estavam matriculadas num jardim de infância da rede pública de ensino, sendo recrutadas seis escolas da região norte e sul de Portugal. As escolas participantes foram selecionadas por conveniência em dois concelhos distintos. O primeiro totalizou cinco escolas com 92 crianças participantes. Destas, 72 crianças foram selecionadas em quatro escolas públicas e 20 crianças numa fundação filantrópica. As escolas deste distrito estavam distribuídas em diferentes freguesias na região do Minho ao norte de Portugal. $\mathrm{O}$ segundo concelho abrangeu uma escola filantrópica pertencente à área metropolitana de Lisboa com 18 crianças participantes. Ambos os concelhos eram predominantemente urbanos e a economia era baseada no setor terciário no período da presente pesquisa. Foram adotados como critérios de exclusão a presença de deficiência sensorial não corrigida ou de necessidade educacional especial, com base em informações fornecidas pela escola, bem como não possuir a língua portuguesa como língua materna. Face ao primeiro critério, foram excluídas três crianças e face ao segundo foram excluídas duas crianças. Após as exclusões, a amostra final foi composta por 105 crianças com idade média de 5.34 ( $D P=.48)$, sendo $60 \%$ do sexo masculino, divididas em dois grupos etários: o grupo de 5 anos foi composto por 69 crianças $(62.3 \%$ o sexo masculino) e o grupo de 6 anos incluiu 36 crianças (55.6\% do sexo masculino).

\section{Instrumentos}

Teste de Atenção por Cancelamento (TAC; Montiel \& Seabra, 2012). O TAC avalia os aspectos de seletividade e alternância atencional. É formado por três partes, cada uma com uma matriz impressa com diferentes tipos de formas geométricas. Em cada parte, a tarefa é assinalar todos os estímulos iguais a um estímulo-alvo. $\mathrm{Na}$ primeira parte, o estímulo-alvo é uma única figura geométrica. Na segunda parte, o estímulo alvo é composto por figuras duplas. Ambas as partes, 1 e 2, avaliam a atenção seletiva. A terceira parte avalia atenção seletiva e alternância, pois o estímulo-alvo muda a cada linha da matriz. O tempo de execução é de um minuto para cada parte. O score utilizado foi o número de acertos (número de alvos corretamente cancelados) em cada parte e no total do TAC. Evidências de validade e dados normativos para a amostra brasileira constam em Seabra e Dias (2012). Exemplos da tarefa em cada parte do teste podem ser consultadas no Material complementar (Figura $1)$.

Teste de Trilhas para Pré-escolares (TT-PE, Trevisan \& Seabra, 2012). O TT-PE avalia a flexibilidade cognitiva. Baseia-se na versão original do Trail Making Test (Partington, Leiter, 1949, adaptação de Montiel \& Capovilla, 2006); contudo, os estímulos originais (letras e números) são neste caso substituídos por figuras, de modo a não exigir conhecimento académico formal. $\mathrm{O}$ teste divide-se em duas partes. Na parte $\mathrm{A}$, é dada à criança uma folha com figuras de cinco cachorrinhos que devem ser ligados por ordem de tamanho. Esta parte mobiliza competências de atenção, busca visual e velocidade. Na parte $\mathrm{B}$, introduzem-se figuras de ossos de tamanhos respectivos aos dos cachorros e a criança deve combinar os cachorrinhos com os seus ossos apropriados, na ordem de tamanho, ligando-os alternadamente. Esta parte do teste, para além de atenção, busca visual e velocidade, exige também flexibilidade cognitiva. Não existe um tempo-limite para a resolução, podendo o tempo decorrido ser anotado para posterior análise. Como medidas de desempenho, utilizaram-se o número de sequências corretas em cada parte, A e B, do teste. A aplicação é individual, com duração média de 7 minutos. Evidências de validade e dados normativos para crianças pré-escolares brasileiras estão publicadas em Seabra e Dias (2012). 
Exemplos da tarefa em cada parte do instrumento podem ser consultadas no Material complementar (Figura 2).

\section{Procedimento}

Apesar de ambos os instrumentos serem nãoverbais, ou seja, não requerem linguagem para a sua resolução, foi realizada a adaptação das instruções do TAC e do TT-PE para o português europeu. Assim, uma análise das instruções foi realizada de modo a minimizar diferenças culturais e de linguagem. Inicialmente, uma análise técnica da linguagem foi realizada por uma profissional nativa de Portugal, com experiência no trabalho com crianças em idade pré-escolar, coordenadora pedagógica de uma escola da rede municipal de ensino de Portugal, com o objetivo de identificar aspectos necessários para a adaptação das instruções. A partir desta primeira análise, foram realizadas algumas adaptações linguísticas, dando origem à primeira versão dos testes para o português europeu.

A partir desta versão, e com apoio da versão original, foi realizada uma análise linguística e semântica por uma especialista em educação com conhecimento de psicometria e avaliação psicológica, pós-doutoranda pelo Centro de Investigações em Ciências da Educação de uma universidade de Portugal. Feito o comparativo qualitativo entre os apontamentos das duas análises, foi elaborada a versão final dos testes para o português europeu, a qual foi utilizada na recolha de dados subsequente.

$\mathrm{O}$ projeto foi submetido e aprovado pelo Comitê de Ética em Pesquisa. Após a apresentação da pesquisa e devida autorização dos diretores regionais, foram indicadas as escolas participantes no estudo. Posteriormente, as escolas foram contatadas e autorizaram a condução da pesquisa. Após a autorização dos responsáveis pelas crianças participantes, mediante assinatura do Termo de Consentimento, iniciou-se a aplicação dos instrumentos. A avaliação das crianças ocorreu nas próprias escolas, individualmente, em uma única sessão de aproximadamente 15 minutos.

\section{Análises estatísticas}

Para verificar o efeito da idade e do sexo sobre os desempenhos nos testes foi conduzida uma análise multivariada (MANOVA), tendo a idade e o sexo como fatores (variáveis independentes) e as diversas medidas (acertos em cada parte e no total do TAC e scores em sequências em cada parte, A e B, do TT-PE) como variáveis dependentes. Calculou-se também o d de Cohen, como estimativa do tamanho do efeito, sendo que valores de $.02, .13$ e .26 representam efeitos pequenos, médios e grandes, respetivamente (Cohen, 1988). Foram conduzidas análises de correlação de Pearson para investigar as relações entre desempenhos em medidas de um mesmo teste e entre desempenhos em medidas dos dois instrumentos. Para comparar os desempenhos da amostra portuguesa com dados normativos das crianças brasileiras foi utilizado o teste nãoparamétrico de Wilcoxon (informações sobre amostra de normalização brasileira disponíveis em Seabra \& Dias, 2012). Para complementar esta análise, calculou-se o tamanho de efeito (d) para testes não-paramétricos. Dados normativos preliminares para as crianças portuguesas foram calculados a partir da fórmula para obtenção de Pontuação-Padrão (PP): $\mathrm{PP}=\{[($ Pontuação bruta média da distribuição)/desvio padrão] x 15$\}+$ 100.

\section{Resultados}

O Quadro 1 apresenta estatísticas descritivas e inferenciais obtidas após MANOVA do efeito da idade (5 e seis anos) e sexo (masculino e feminino) sobre os desempenhos no TAC e TTPE. Houve efeito da idade sobre todas as medidas do TAC, com tamanhos de efeito entre pequeno e moderado e melhor desempenho das crianças mais velhas. Para o TT-PE, apesar da tendência de melhor desempenho com a progressão da idade, não houve diferença significativa entre os grupos e os tamanhos de efeito foram pequenos. $\mathrm{O}$ efeito do sexo foi observado somente sobre o número de acertos na parte 1 e total do TAC, com tamanho de efeito moderado e melhor desempenho das meninas. Não houve efeito da interação idadesexo sobre as medidas utilizadas.

A análise das correlações de Pearson revelou diversas correlações significativas, tal como observado no Quadro 2. A correlação entre os desempenhos nas partes A e B do TT-PE foi apenas de magnitude baixa, apesar de significativa. 
Quadro 1. Estatísticas Descritivas e Inferenciais Obtidas Após MANOVA (Idade x Sexo) sobre os Desempenhos no TT-PE e TAC

\begin{tabular}{|c|c|c|c|c|c|c|c|c|c|c|c|c|c|}
\hline \multirow[b]{4}{*}{$\begin{array}{l}\text { TT_PE } \\
\text { parte A }\end{array}$} & \multicolumn{9}{|c|}{ Média $(D P)$} & \multirow{2}{*}{\multicolumn{2}{|c|}{$\begin{array}{l}\text { Efeito da } \\
\text { idade }\end{array}$}} & \multirow{2}{*}{\multicolumn{2}{|c|}{$\begin{array}{l}\text { Efeito do } \\
\text { sexo }\end{array}$}} \\
\hline & \multicolumn{3}{|c|}{ Grupo 5 anos } & \multicolumn{3}{|c|}{ Grupo 6 anos } & \multicolumn{3}{|c|}{ Amostra total } & & & & \\
\hline & M & $\mathrm{F}$ & Total & M & $\mathrm{F}$ & Total & M & F & Total & $\begin{array}{c}F \\
(1,101)\end{array}$ & $d$ & $\begin{array}{c}F \\
(1,101)\end{array}$ & $d$ \\
\hline & $\begin{array}{c}3.60 \\
(1.81)\end{array}$ & $\begin{array}{c}3.81 \\
(1.67)\end{array}$ & $\begin{array}{c}3.68 \\
(1.75)\end{array}$ & $\begin{array}{c}3.85 \\
(1.46)\end{array}$ & $\begin{array}{c}4.19 \\
(1.56)\end{array}$ & $\begin{array}{c}4.00 \\
(1.49)\end{array}$ & $\begin{array}{c}3.68 \\
(1.70)\end{array}$ & $\begin{array}{c}3.95 \\
(1.62)\end{array}$ & $\begin{array}{c}3.79 \\
(1.67)\end{array}$ & .794 & .192 & .594 & .163 \\
\hline $\begin{array}{l}\text { TT_PE } \\
\text { parte B }\end{array}$ & $\begin{array}{l}2.91 \\
(2.23)\end{array}$ & $\begin{array}{c}2.96 \\
(2.22)\end{array}$ & $\begin{array}{c}2.93 \\
(2.23)\end{array}$ & $\begin{array}{c}3.20 \\
(2.48)\end{array}$ & $\begin{array}{c}3.75 \\
(2.24)\end{array}$ & $\begin{array}{c}3.44 \\
(2.36)\end{array}$ & $\begin{array}{l}3.00 \\
(2.32)\end{array}$ & $\begin{array}{c}3.26 \\
(2.23)\end{array}$ & $\begin{array}{c}3.10 \\
(2.28)\end{array}$ & 1.277 & .224 & .399 & .114 \\
\hline $\mathrm{TAC}-1$ & $\begin{array}{c}28.70 \\
(10.31)\end{array}$ & $\begin{array}{l}33.00 \\
(8.94)\end{array}$ & $\begin{array}{l}30.32 \\
(9.97)\end{array}$ & $\begin{array}{l}33.80 \\
(8.84)\end{array}$ & $\begin{array}{l}38.44 \\
(7.13)\end{array}$ & $\begin{array}{l}35.86 \\
(8.35)\end{array}$ & $\begin{array}{c}30.32 \\
(10.08)\end{array}$ & $\begin{array}{l}35.07 \\
(8.63)\end{array}$ & $\begin{array}{l}32.22 \\
(9.77)\end{array}$ & $\begin{array}{c}7.397 \\
* *\end{array}$ & .586 & $\begin{array}{c}5.322 \\
*\end{array}$ & .506 \\
\hline $\mathrm{TAC}-2$ & $\begin{array}{c}1.33 \\
(1.27)\end{array}$ & $\begin{array}{l}1.35 \\
(1.06)\end{array}$ & $\begin{array}{c}1.33 \\
(1.18)\end{array}$ & $\begin{array}{c}1.60 \\
(0.99)\end{array}$ & $\begin{array}{c}2.06 \\
(1.29)\end{array}$ & $\begin{array}{c}1.81 \\
(1.14)\end{array}$ & $\begin{array}{c}1.41 \\
(1.19)\end{array}$ & $\begin{array}{c}1.62 \\
(1.19)\end{array}$ & $\begin{array}{c}1.50 \\
(1.19)\end{array}$ & $\begin{array}{c}4.092 \\
*\end{array}$ & .41 & .973 & .176 \\
\hline $\mathrm{TAC}-3$ & $\begin{array}{l}12.02 \\
(5.56)\end{array}$ & $\begin{array}{l}12.35 \\
(5.95)\end{array}$ & $\begin{array}{l}12.14 \\
(5.67)\end{array}$ & $\begin{array}{l}14.40 \\
(7.00)\end{array}$ & $\begin{array}{l}16.38 \\
(3.81)\end{array}$ & $\begin{array}{l}15.28 \\
(5.81)\end{array}$ & $\begin{array}{l}12.78 \\
(6.10)\end{array}$ & $\begin{array}{l}13.88 \\
(5.55)\end{array}$ & $\begin{array}{l}13.22 \\
(5.89)\end{array}$ & $\begin{array}{c}7.136 \\
* *\end{array}$ & .549 & .918 & .189 \\
\hline $\begin{array}{l}\text { TAC - } \\
\text { total }\end{array}$ & $\begin{array}{c}42.05 \\
(14.43)\end{array}$ & $\begin{array}{c}46.69 \\
(13.09)\end{array}$ & $\begin{array}{c}43.80 \\
(14.03)\end{array}$ & $\begin{array}{c}49.80 \\
(14.59)\end{array}$ & $\begin{array}{l}56.88 \\
(8.88)\end{array}$ & $\begin{array}{c}52.94 \\
(12.73)\end{array}$ & $\begin{array}{c}44.51 \\
(14.82)\end{array}$ & $\begin{array}{c}50.57 \\
(12.59)\end{array}$ & $\begin{array}{c}46.93 \\
(14.22)\end{array}$ & $\begin{array}{c}10.214 \\
* *\end{array}$ & .672 & $\begin{array}{c}4.361 \\
*\end{array}$ & .441 \\
\hline
\end{tabular}

Quadro 2. Matriz de Correlações entre os Scores da TAC e TT-PE

\begin{tabular}{lcccccc}
\hline & & $\begin{array}{c}\text { TT_PE }- \\
\text { parte A }\end{array}$ & $\begin{array}{c}\text { TT_PE }- \\
\text { parte B }\end{array}$ & TAC -1 & TAC - 2 & TAC - 3 \\
\hline TT_PE - parte B & $r$ & .32 & & & & \\
& $p$ & .001 & & & & \\
TAC - 1 & $r$ & .25 & .33 & & & \\
& $p$ & .009 & .001 & & & \\
TAC -2 & $r$ & .25 & .30 & .30 & & \\
& $p$ & .009 & .002 & .002 & & \\
TAC - 3 & $r$ & .46 & .36 & .52 & .28 & \\
& $p$ & $<.001$ & $<.001$ & $<.001$ & .004 & \\
TAC - total & $r$ & .39 & .40 & .93 & .41 & .80 \\
& $p$ & $<.001$ & $<.001$ & $<.001$ & $<.001$ & $<.001$ \\
\hline
\end{tabular}

Quadro 3. Resultado da Comparação entre Dados das Crianças Portuguesas e Amostra de Normalização Brasileira

\begin{tabular}{lccccccc}
\hline Idade & & \multirow{2}{*}{$\mathrm{TAC}-1$} & $\mathrm{TAC}-2$ & $\mathrm{TAC}-3$ & \multirow{2}{*}{$\mathrm{TAC}-$ total } & $\begin{array}{c}\text { TT-PE }- \\
\text { Parte A }\end{array}$ & $\begin{array}{c}\text { TT-PE }- \\
\text { Parte B }\end{array}$ \\
\hline \multirow{2}{*}{5} & $\mathrm{Z}$ & -4.939 & -2.311 & -2.840 & -4.813 & 4.710 & 2.689 \\
& $p$ & $<.001$ & .021 & .005 & $<.001$ & $<.001$ & .007 \\
& $d$ & 1.479 & .579 & .728 & 1.422 & 1.377 & .684 \\
6 & $\mathrm{Z}$ & -3.845 & -3.522 & -.534 & -2.998 & 3.247 & -.134 \\
6 & $p$ & $<.001$ & $<.001$ & .594 & .003 & .001 & .893 \\
& $d$ & 1.67 & 1.45 & .179 & 1.154 & 1.287 & .045 \\
\hline
\end{tabular}

Entre as medidas do TAC, as correlações foram de baixas a altas. Já entre os dois instrumentos, foram de baixas a moderadas. Estes resultados sugerem exigências específicas de cada parte e de ambos os testes. Por outro lado, considerando o total de acertos no TAC, as relações com as diferentes partes do instrumento variaram de moderada a alta, evidenciando relativa consistência interna.

Observaram-se diferenças significativas entre o desempenho das crianças portuguesas e da amostra de normalização brasileira na maioria das medidas de ambos os instrumentos, com tamanhos de efeito entre moderado e grande, conforme apresentado no Quadro 3. Diferenças entre os desempenhos das amostras apenas não foram observadas para o TAC - parte 3 e TT-PE - parte B, ambos na amostra de 6 anos, com tamanhos de efeito pequenos. As crianças portuguesas apresentaram melhor desempenho nas medidas do TAC em relação à amostra de normalização brasileira. Por sua vez, as crianças brasileiras tiveram melhor desempenho no TT-PE, partes A e B.

Face às diferenças observadas, optou-se pelo cálculo de dados normativos preliminares para a amostra portuguesa. Os Quadros com a pontuação-padrão para crianças portuguesas de 5 e 6 anos para as medidas do TT-PE e TAC podem ser obtidas no Material Suplementar ou em contato com a primeira autora deste artigo. 


\section{Discussão}

Este estudo realizou a adaptação das instruções do Teste de Atenção por Cancelamento e Teste de Trilhas para crianças em idade préescolar para o português europeu (disponíveis no Material Suplementar ou a partir de contato com a primeira autora). Além disso, teve como objetivo investigar evidências de validade de ambos os instrumentos e disponibilizar dados normativos preliminares para crianças portuguesas em idade pré-escolar.

Os resultados apontam no sentido de um desempenho crescente na tarefa de atenção, com maior número de acertos no TAC no grupo de 6 anos em relação ao grupo de 5 anos. Contudo, no caso do TT-PE não foram observadas diferenças de desempenho entre os grupos etários. Com efeito, a literatura tem apontado para diferentes trajetórias de desenvolvimento para as distintas FE. Assim, a habilidade de focalizar a atenção parece se desenvolver mais precocemente, com alguns autores elencando a idade entre os 3 e 5 anos como de rápido desenvolvimento desta habilidade (Best \& Miller, 2010) e outros destacando que, aos 7 anos, as crianças já seriam mais eficazes em lidar com distratores e focalizar a atenção (CDCHU, 2011). Neste sentido, o instrumento utilizado parece ter sido discriminativo para detectar essa diferença de desempenho entre os 5 e 6 anos de idade.

Por outro lado, os resultados sugerem que a flexibilidade cognitiva pode desenvolver-se mais tardiamente. Por exemplo, para Diamond (2013) a flexibilidade desenvolver-se-ia mais tarde por ser uma habilidade mais complexa, na medida em que envolveria em algum grau a inibição e a memória de trabalho. Autores como Best e Miller (2010) destacam que entre os 5 e 7 anos haveria uma importante progressão da flexibilidade. Porém, outros sugerem que esta habilidade continuaria a se desenvolver de forma mais intensa até aproximadamente os 12 anos de idade, continuando ao longo da adolescência (CDCHU, 2011). Neste sentido, é possível que o desenvolvimento desta habilidade ocorra de forma mais gradativa, não necessariamente linear, no recorte específico incluído neste estudo, de modo que diferenças entre grupos etários próximos não sejam tão evidentes. Com efeito, apesar da ausência de significância estatística, os resultados das análises descritivas mostraram uma tendência para um melhor desempenho das crianças de 6 anos em comparação com os seus pares mais jovens. Resultados semelhantes foram observados num estudo brasileiro, em que as medidas do TT$\mathrm{PE}$ discriminaram as crianças da fase $1 \mathrm{da}$ Educação Infantil (idade média de 4 anos) em relação às crianças da fase 2 ( 5 anos) e do $1^{\circ}$ ano do Ensino Fundamental (6 anos), mas não discriminaram os últimos níveis escolares entre si (Trevisan, 2010). Sumariamente, os resultados deste estudo fornecem evidências de validade por mudança no desenvolvimento ao TAC. No caso do TT-PE, parece ser o caso de que a habilidade mensurada se desenvolva de modo mais gradual nesta faixa etária, de modo que o instrumento pode não ter sido sensível para detectar os ganhos entre os 5 e os 6 anos de idade.

Foram observadas diferenças entre crianças do sexo masculino e feminino, com melhor desempenho das meninas, apenas para a parte $1 \mathrm{e}$, como possível reflexo disto, no total de acertos do TAC. Não foi observado efeito do sexo nas demais medidas. Efetivamente, pelo menos na primeira e segunda infância, o efeito do sexo parece não ocorrer em tarefas executivas. Por exemplo, Moura et al. (2013) não encontraram efeito do sexo em tarefas de fluência verbal e também Teixeira (2012) não observou efeito em tarefa de inibição e flexibilidade. Os resultados de Pinto (2008), embora mais inconsistentes, também apontam nessa direção, visto que a autora encontrou efeito do sexo, com melhor desempenho para as meninas, apenas em uma tarefa de memória de trabalho auditiva, apesar de não o ter notado em outros instrumentos, que incluíam testes de inibição, flexibilidade, fluência e planeamento. Os resultados deste estudo parecem bastante alinhados com essa literatura, de modo que não se julgou necessária a provisão de dados normativos divididos por sexo.

As correlações entre os instrumentos revelaram boa consistência interna para o TAC, considerando as relações entre desempenho em cada uma das partes do instrumento com o score total. Por outro lado, as relações entre as partes do teste, o que também ocorreu com o TT-PE, mostraram que essas avaliam aspectos relativamente distintos, com exigências 
específicas. Com efeito, no caso do TAC, as partes 1 e 2 avaliam seletividade, porém com níveis de complexidade distintos, enquanto a parte 3 apresenta uma exigência de alternância (Seabra \& Dias, 2012). No caso do TT-PE, a parte A avalia sequenciamento e busca visual, enquanto que a parte $B$ inclui exigência de flexibilidade (Trevisan, 2010). As relações entre os índices dos instrumentos mostraram alguma associação, porém evidência de relativa independência entre os construtos, corroborando os componentes de inibição (atenção seletiva) e flexibilidade do modelo de Diamond (2013). Resultados semelhantes foram observados em estudos com crianças em idade pré-escolar no Brasil, com relações em geral baixas entre o TAC e o TT-PE (Seabra \& Dias, 2012). Apesar de esperarmos maior associação entre as medidas (cf. Brydges et al., 2014; Garon et al., 2008), esses resultados podem sugerir que já há relativa dissociação entre essas habilidades em crianças entre os 5 e 6 anos de idade.

No tocante à comparação do desempenho das crianças portuguesas com os dados normativos das crianças brasileiras, foram observadas diferenças de desempenho, sendo que as crianças portuguesas tiveram melhor desempenho em todas as medidas do TAC (exceto na parte 3 para as crianças de 6 anos), denotando um melhor desenvolvimento da atenção na amostra portuguesa. Por outro lado, a amostra brasileira manteve melhor desempenho no TT-PE, demonstrando melhor busca visual (parte A do TT-PE) e mesmo melhor flexibilidade cognitiva (parte B) no grupo de 5 anos em comparação com as crianças portuguesas, apesar de uma tendência a esse desempenho se igualar no grupo de 6 anos. Estes resultados corroboram estudos prévios que têm destacado o papel da cultura (Carlson, 2009) e de outras variáveis ambientais (Engel de Abreu et al., 2015; Hammond et al., 2012; Noble et al., 2015) no desenvolvimento das FE. É possível que as culturas brasileira e portuguesa enfatizem diferentemente tais habilidades, influenciando o seu desenvolvimento. Contudo, é interessante notar que aos 6 anos os desempenhos das amostras tendem a equilibrar-se nos índices mais complexos de ambos os testes, isto é, parte 3 do TAC e parte B do TT-PE. Isto pode refletir aspectos maturacionais das funções avaliadas.
Contudo, uma explicação para este resultado permanece em aberto.

Face às diferenças observadas e procurando responder ao segundo objetivo do estudo, foram disponibilizados dados normativos preliminares para crianças portuguesas de 5 e 6 anos (acesso no Material suplementar ou via contato com a primeira autora). Ao prover algumas evidências de validade e dados normativos preliminares, o estudo contribui para instrumentalizar a área de avaliação de FE na infância, sobretudo na etapa pré-escolar (Barros \& Hazin, 2013) e soma os seus esforços a estudos portugueses na área (e.g., Moura et al., 2013; Moura et al., 2017; Pinto, 2008; Ribeiro et al., 2012; Simões et al., 2016; Teixeira, 2012). Reconhecendo a associação e contribuição das FE para o comportamento, aprendizagem e saúde mental infantil (Dias et al., 2017; Nelson et al., 2018; Nesbitt et al., 2015; Seabra et al., 2014), além de seu comprometimento nos Transtornos do Neurodesenvolvimento (Kimhi et al., 2014; PauliPott \& Becker, 2011), é importante que profissionais nos contextos clínico e escolar considerem a avaliação precoce dessas habilidades, não só para fins diagnósticos, mas para mapeamento de áreas de dificuldade. Esse será o primeiro passo para o planeamento de programas de estimulação.

$\mathrm{O}$ estudo inclui entre as suas limitações o pequeno número amostral e a ausência de investigação de outras fontes de evidência de validade, como padrões de convergência com outros instrumentos. Por outro lado, a amostra do presente estudo foi muito restrita em termos de faixa etária. No entanto, o estudo é um primeiro passo para disponibilizar no país mais duas opções para a avaliação infantil, sem descartar a necessidade de novas investigações, entre elas de evidências de validade convergente com subtestes da BANC e mesmo estudos transculturais acerca do desenvolvimento das FE (que podem ser facilitados a partir do uso das mesmas medidas). Dada a relevância destas habilidades (e.g., CDCHU, 2011; Diamond, 2013; Engel de Abreu et al., 2015), a sua avaliação na infância pode ser importante para a identificação de atrasos no desenvolvimento, de modo que intervenções possam ser dirigidas precocemente. Para tal, torna-se necessário a instrumentalização dos 
profissionais da área.

\section{Considerações Finais}

$\mathrm{O}$ presente estudo forneceu algumas evidências de validade de dois instrumentos de avaliação de habilidades das $\mathrm{FE}$ em crianças portuguesas em idade pré-escolar. A partir da comparação do desempenho da amostra portuguesa com dados normativos do Brasil, forneceu também dados para uma normalização preliminar, para uso de investigadores e profissionais na avaliação de crianças de 5 e 6 anos de idade em Portugal. Neste sentido, o estudo espera contribuir para a área da avaliação neuropsicológica infantil.

\section{Referências}

Barros, P. M., \& Hazin, I. (2013). Avaliação das funções executivas na infância: Revisão dos conceitos e instrumentos. Psicologia em Pesquisa, 7(1), 13-22. doi:10.5327/Z1982-1247201300010003

Bártolo-Ribeiro, R. Mário R. Simões, M. R., \& Almeida, L. S. (2016). Metacognitive Awareness Inventory (MAI): Adaptação e validação da versão portuguesa. Revista Iberoamericana de Diagnóstico y Evaluación - e Avaliação Psicológica, 42(2), 143-159. doi:10.21865/RIDEP42_145

Bernier, A., Carlson, S. M., Deschênes, M., \& Matte-Gagné, C. (2012). Social factors in the development of early executive functioning: A closer look at the caregiving environment. Developmental Science, 15(1), 12-24. doi:10.1111/j.1467-7687.2011.01093.x

Best, J. R., \& Miller, P. H. (2010). A developmental perspective on executive function. Child Development, 81(6), 16411660. doi:10.1111/j.1467-8624.2010.01499.x

Bierman, K. L., \& Torres, M. (2016). Promoting the development of executive functions through early education and prevention programs. In J. A. Griffin, L. S. Freund, \& P. McCardle (Eds.), Executive function in preschool age children: Integrating measurement, neurodevelopment and translational research. Washington, DC: APA.

Blair, C. (2016). Executive function and early childhood education. Current Opinion in Behavioral Sciences, 10, 1-6. doi:10.1016/j.cobeha.2016.05.009

Brydges, C. R., Fox, A. M., Reid, C. L., \& Anderson, M. (2014). The differentiation of executive functions in middle and late childhood: A longitudinal latent-variable analysis. Intelligence, 47, 34-43. doi: 10.1016/j.intell.2014.08.010

Campbell, F. A., Pungello, E. P., Miller-Johnson, S., Burchinal, M., \& Ramey, C. T. (2001). The development of cognitive and academic abilities: Growth curves from an early childhood educational experiment. Developmental Psychology, 37(2), 231-42. doi:10.1037/0012-1649.37.2.231

Carapito, E., Ribeiro, M. T., \& Pereira, A. I. (2015). Questionário de Envolvimento Parental no Jardim-de-Infância (QEPJI): Estudo de validação da versão para pais. Revista Iberoamericana de Diagnóstico y Evaluación - e Avaliação Psicológica, 40(2), 83-93. Recuperado de: http://www.aidep.org/sites/default/files/article s/R40/Art8.pdf

Carlson, S. M. (2009). Social origins of executive function development. In C. Lewis \& J. I. M. Carpendale (Eds.), Social interaction and the development of executive function. New directions in child and adolescent development, 123 (pp. 87-97). doi:10.1002/cd.237

Center on the Developing Child at Harvard University. (2011). Building the brain's "air trafic control" system: How early experiences shape the development of executive function. Relatório técnico. http://developingchild.harvard.edu/wpcontent/uploads/2011/05/How-EarlyExperiences-Shape-the-Development-ofExecutive-Function.pdf

Cohen, J. (1988). Statistical power analysis for the behavioral sciences (2nd ed.). Hillsdale, NJ: Lawrence Earlbaum Associates.

Diamond, A. (2013). Executive functions. Annual Review of Psychology, 64, 135-168. doi:10.1146/annurev-psych-113011-143750

Diamond, A. (2014). Executive functions: Insights into ways to help more children thrive. Zero to Three, 9-18. Recuperado de: http://www.devcogneuro.com/Publications 
/zero_to_three_2014_executi-

ve_functions.pdf

Diamond, A., \& Lee, K. (2011). Interventions shown to aid executive function development in children 4 to 12 years old. Science, 333(6045), 959-964. doi:10.1126/science.1204529

Dias, N. M., \& Seabra, A. G. (2013). Funções executivas: Desenvolvimento e intervenção. Temas sobre Desenvolvimento, 19(107), 206$212 . \quad$ Recuperado de: https://www.researchgate.net/publication/281 177320

Dias, N. M., \& Seabra, A. G. (2015). Is it possible to promote executive functions in preschoolers? A case study in Brazil. International Journal of Child Care and Education Policy, 9(6), 1-18. doi:10.1186/s40723-015-0010-2

Dias, N. M., Trevisan, B. T., León, C. B. R., Prust, A. P., \& Seabra, A. G. (2017). Can executive functions predict behavior in preschool children? Psychology \& Neuroscience, 10(4), 383-393. doi: $10.1037 /$ pne0000104

Engel de Abreu, P., Tourinho, C., Puglisi, M., Nikaedo, C., Abreu, N., Miranda, M., BefiLopes, D, Bueno, O., \& Martin, R. (2015). A pobreza e a mente: Perspectiva da ciência cognitiva. Relatório Técnico. University of Luxembourg. Recuperado de: https://orbilu.uni.lu/bitstream/10993/20933/1/ Engel\%20de\%20Abreu\%20et\%20al._Poverty $\% 20$ and $\% 20$ the $\% 20$ Mind.pdf

Espy, K., Bull, R. B., Martin, J., \& Stroup, W. (2006). Measuring the development of executive control with the Shape School. Psychological Assessment, 18(4), 373-81. doi:10.1037/1040-3590.18.4.373

Friedman, N. P., \& Miyake, A. (2017). Unity and diversity of executive functions: Individual differences as a window on cognitive structure. Cortex, 86, 186-204. doi:10.1016/j.cortex.2016.04.023

Garon, N., Bryson, S. E., \& Smith, I. M. (2008). Executive function in preschoolers: A review using an integrative framework. Psychological Bulletin, 134(1), 31-60. doi:10.1037/0033-2909.134.1.31

Hammond, S. I., Müller, U., Carpendale, J. I. M., Bibok, M. B., \& Liebermann-Finestone, D. P.
(2012). The effects of parental scaffolding on preschoolers' executive function. Developmental Psychology, 48(1), 271-81. doi:10.1037/a0025519

Heckman, J. J. (2006). Skill formation and the economics of investing in disadvantaged children. Science, 312(5782), 1900-2.

doi:10.1126/science. 1128898

Kimhi, Y., Shoam-Kugelmas, D., Agam BenArtzi, G., Ben-Moshe, I., \& BaumingerZviely, N. (2014). Theory of mind and executive function in preschoolers with typical development versus intellectually able preschoolers with autism spectrum disorder. Journal of Autism and Developmental Disorders, 44(9), 2341-54. doi:10.1007/s10803-014-2104-z

Llorente, A. M., Williams, J., Satz, P., \& D`Elia, L. F. (2003). Children's Color Trails Test: Professional manual. U.S.A.: Psychological Assessment Resources.

Miyake, A., Friedman, N. P., Emerson, M. J., Witzki, A. H., Howerter, A., \& Wager, T. D. (2000). The unity and diversity of executive functions and their contributions to complex "frontal lobe" tasks: A latent variable analysis. Cognitive Psychology, 41(1), 49100. doi:10.1006/cogp.1999.0734

Montiel, A. G. S. \& Capovilla, F. C. (2006). Teste de Trilhas - Partes A e B. In A. G. S. Capovilla (Ed.), Teoria e pesquisa em avaliação neuropsicológica (pp. 109-114). São Paulo: Memnon.

Montiel, J. M., \& Seabra, A. G. (2012). Teste de atenção por cancelamento. In A. G. Seabra \& N. M. Dias (Eds.), Avaliação neuropsicológica cognitiva: Atenção $e$ funções executivas (pp. 57-66). São Paulo: Memnon.

Moura, O., Albuquerque, C. P., Salomé Pinho, M., Vilar, M., Filipa Lopes, A., Alberto, I., Pereira, M., Santos, M. J. S., \& Simões, M. R. (2017). Factor structure and measurement invariance of the Coimbra Neuropsychological Assessment Battery (BANC). Archives of Clinical Neuropsychology, 1-13. doi:10.1093/arclin/acx052 Moura, O., Simões, M. R., \& Pereira, M. (2013). Fluência verbal semântica e fonêmica em crianças: Funções cognitivas e análise 
temporal. Avaliação Psicológica, 12(2), 167177.

Nelson, T. D., Kidwell, K. M., Nelson, J. M., Tomaso, C. C., Hankey, M., \& Espy, K. A. (2018). Preschool executive control and internalizing symptoms in elementary school. Journal of Abnormal Child Psychology, 1-12. doi:10.1007/s10802-017-0395-1

Nesbitt, K. T., Farran, D. C., \& Fuhs, M. W. (2015). Executive function skills and academic achievement gains in prekindergarten: Contributions of learningrelated behaviors. Developmental Psychology, 51(7), 865-78. doi:10.1037/dev0000021

Noble, K. G., Houston, S. M., Brito, N. H., Bartsch, H., Kan, E., Kuperman, J. M., \& Sowell, E. R. (2015). Family income, parental education and brain structure in children and adolescents. Nature Neuroscience, 18(5), 773778. doi:10.1038/nn.3983

Partington, J. E. \& Leiter, R. G. (1949). Partington's Pathways Test. Journal of Consulting Psychology, 13(5), 386. doi:10.1037/h0049525

Pauli-Potta, U., \& Becker, K. (2011). Neuropsychological basic deficits in preschoolers at risk for ADHD: A metaanalysis. Clinical Psychology Review, 31(4), 626-637. doi:10.1016/j.cpr.2011.02.005

Pinto, A. B. (2008). Desenvolvimento das funções executivas em crianças dos 6 aos 11 anos de idade. Dissertação de Mestrado. Universidade do Porto. Recuperado de: http://hdl.handle.net/10216/106299

Ribeiro, I. G., Cruz-Santos, A., \& MirandaCorreia, L. (2012). Behavior rating inventory of executive functions. Encontro: Revista de Psicologia, 15(22), 25-36. Recuperado de: http://www.pgsskroton.com.br/seer/index.php/ renc/article/view/2478/2374

Schraw, G., \& Dennison, R. S. (1994). Assessing metacognitive awareness. Contemporary Educational Psychology, 19, 460-475. doi:10.1006/ceps.1994.1033

Seabra, A. G., \& Dias, N. M. (2012). Avaliação neuropsicológica cognitiva: Atenção $e$ funções executivas (1st ed.). São Paulo: Memnon.

Seabra, A. G., Muniz, M., Reppold, C. T., Dias, N. M., Siquara, G., Tourinho, A. M. O.,
Gurgel, L. G., Teixeira, L. P. (2014). Funções executivas e desempenho escolar. In A. G. Seabra, J. A. Laros, E. C. Macedo, \& J. N. Abreu (Eds.), Inteligência e funções executivas: Avanços e desafios para a avaliação neuropsicológica (pp. 211-225). São Paulo: Memnon.

Simões, M. R., Albuquerque, C. P., Pinho, M. S., Pereira, M., Seabra-Santos, M. J., Alberto, I., \& Lopes, A. F. (2016). Bateria de Avaliação Neuropsicológica de Coimbra $(B A N C)$. Lisboa: Cegoc.

Teixeira, S. C. M. (2012). Funções executivas e competência linguísticas em crianças dos 3 aos 4 anos. Monografia. Universidade Atlântica. Recuperado de: https://repositoriocientifico.uatlantica.pt/bitstr eam/10884/823/2/Monografia\%20Sara\%2020 $0891782 \% 20$

\%20Vers\%C3\%A3o\%20Final\%20Corrigida.p df

Trevisan, B. T. (2010). Atenção e controle inibitório em pré-escolares e correlação com indicadores de desatenção e hiperatividade. Dissertação de Mestrado. Universidade Presbiteriana Mackenzie. Recuperado de: http://tede.mackenzie.br/jspui/handle/tede/153 7

Trevisan, B. T., \& Seabra, A. G. (2012). Teste de trilhas para pré-escolares. In A. G. Seabra \& N. M. Dias (Eds.), Avaliação neuropsicológica cognitiva: Atenção $e$ funções executivas (pp. 92-100). São Paulo: Memnon.

Wiebe, S. A., Sheffield, T., Nelson, J. M., Clark, C. A. C., Chevalier, N., \& Espy, K. A. (2011). The structure of executive function in 3-yearolds. Journal of Experimental Child Psychology, 108(3), 436-452. doi:10.1016/j.jecp.2010.08.008 


\section{Material Suplementar}

Exemplos das tarefas solicitadas nos instrumentos utilizados

Teste de Atenção por Cancelamento (TAC)

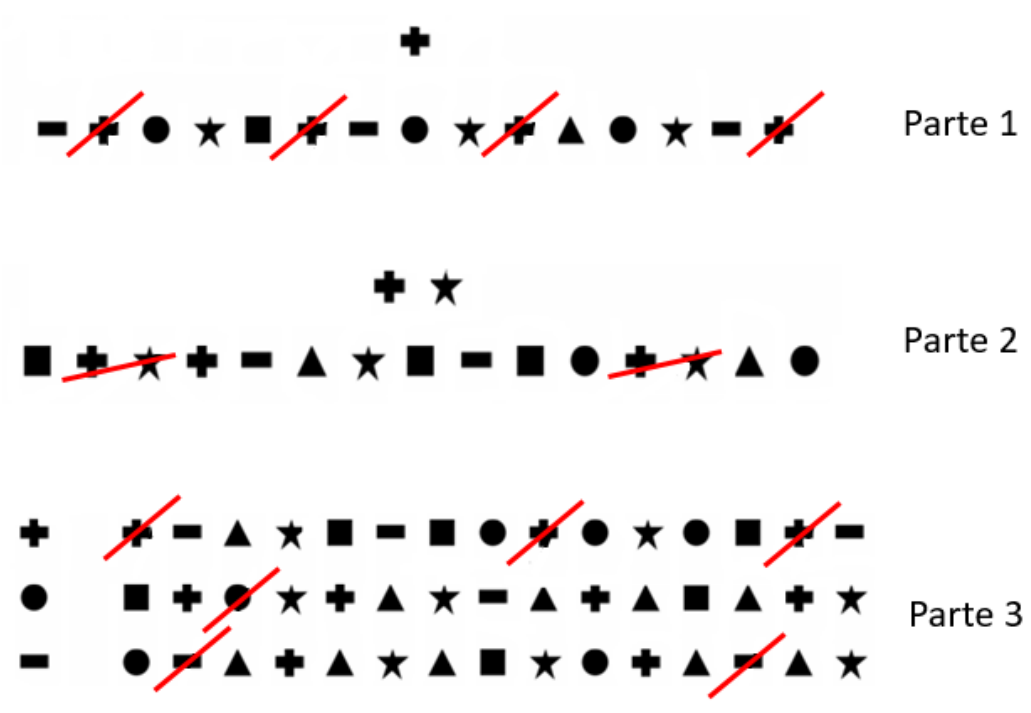

Figura 1. Exemplos da tarefa requerida em cada parte do TAC

Teste de Trilhas para pré-escolares (TT-PE)

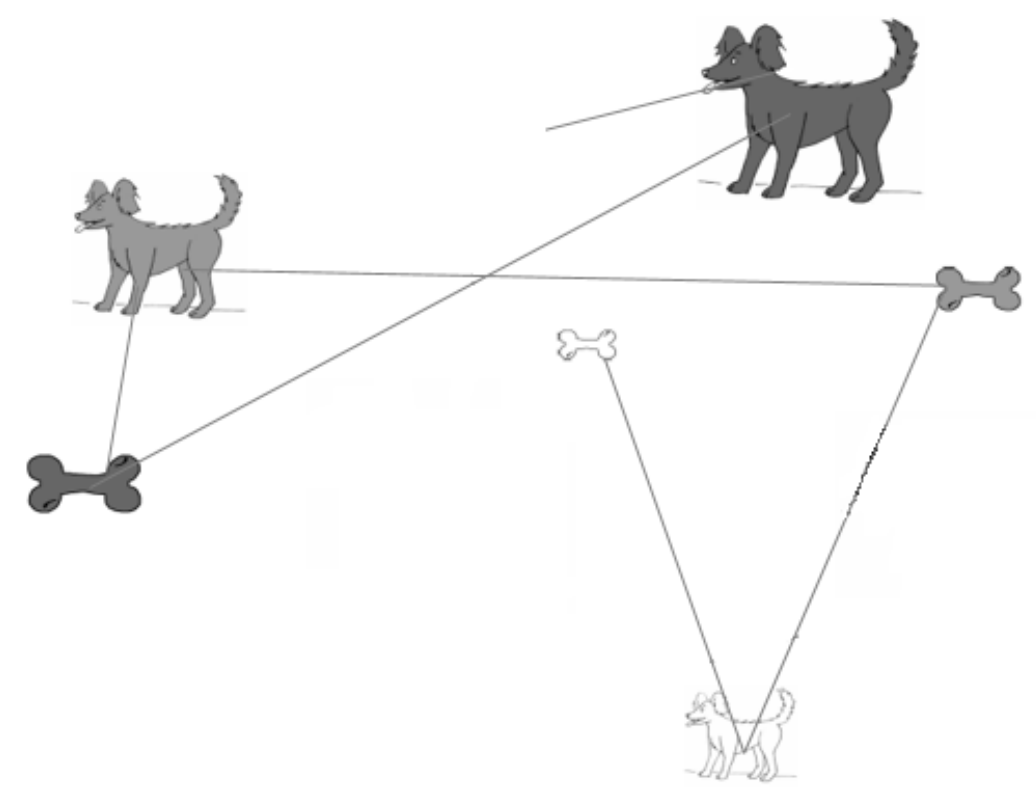

Figura 2. Exemplo da tarefa requerida na parte B do TT-PE

\section{Instruções adaptadas para o português de Portugal}

Teste de Atenção por Cancelamento

\section{Parte 1 - Instruções}

Vai fazer um teste de atenção com três fases. Veja o exemplo abaixo para fazer a primeira fase do teste. Há uma figura na parte de cima (uma cruz) e uma sequência com várias figuras na parte de baixo (quadrados, círculos, triângulos, retângulos, estrelas e cruzes).

Observe que, na sequência de figuras, foram cortadas aquelas que são iguais à figura da parte de cima. 


\section{$<$ Exemplo>}

$\mathrm{Na}$ folha seguinte encontra uma outra figura na parte de cima e uma outra sequência na parte de baixo. Como no exemplo, procure e corte as figuras iguais à figura da parte de cima.

Tem 1 minuto para realizar a atividade. Faça o mais rápido que conseguir.

$<$ Folha de Treino>

<Início da aplicação da parte 1 do teste>

\section{Parte 2 - Instruções}

Nesta segunda fase, encontra duas figuras na parte de cima da folha, como no exemplo abaixo.

Observe que, na sequência de figuras, foram cortados os pares que são iguais ao da parte de cima.

\section{$<$ Exemplo>}

$\mathrm{Na}$ folha seguinte encontra outras duas figuras na parte de cima e uma outra sequência na parte de baixo. Como no exemplo, procure e corte os pares de figuras que forem iguais ao da parte de cima.

Lembre-se que deve cortar somente os pares exatamente iguais ao modelo, ou seja, os pares que estiverem na mesma ordem. Tem também 1 minuto para realizar a atividade. Faça o mais rápido que conseguir.

$<$ Folha de Treino>

<Início da aplicação da parte 2 do teste>

\section{Parte 3 - Instruções}

Esta é a terceira e última fase do teste. Veja o exemplo abaixo.

Há uma figura no início de cada linha e uma sequência com várias figuras (quadrados, círculos, triângulos, retângulos, estrelas e cruzes).

Foram cortadas, em cada linha, as figuras que são iguais à primeira figura da fila.

\section{$<$ Exemplo>}

$\mathrm{Na}$ folha seguinte encontra outras linhas, sempre com uma figura inicial e uma sequência de figuras. Como no exemplo, procure e corte as figuras que forem iguais à primeira figura de cada fila.

Tem 1 minuto para fazer a atividade. Faça o mais rápido que conseguir.

$<$ Folha de Treino>

<Início da aplicação da parte 3 do teste>

\section{Teste de Trilhas para pré-escolares}

\section{Parte A Folha de instrução 1}

Era uma vez uma família de cãezinhos. Faziam parte da família: o pai, a mãe, o filho mais velho, o filho do meio e o filho mais novo. Veja a família de cãezinhos: o pai é muito grande; a mãe é um pouquinho mais pequena que o pai; o filho mais velho é um pouco mais pequeno que a mãe; o filho do meio é mais pequeno que o filho mais velho; e finalmente, o filho mais novo, é o mais pequeno de todos.

<figuras da família de cachorros>

FILHO MAIS NOVO

FILHO DO MEIO

FILHO MAIS VELHO

MÃE

PAI

\section{Parte A Folha de instrução 2}

Agora, a família de cãezinhos precisa ir para casa. Então, todos os cãezinhos precisam ir juntos. Para isso, precisamos unir todos eles. No entanto, precisamos uni-los por ordem de tamanho. Vamos ligar os cãezinhos começando pelo filho mais novo, seguido do filho do meio, do filho mais velho, da mãe e do pai. Sem tirar o lápis do papel! Veja o exemplo.

$<$ Exemplo>

<Início da aplicação da parte A do teste>

Parte B Folha de instrução 1 
Agora, os cãezinhos estão com fome. Então, antes de ir para casa juntos, eles precisam comer. Sabe o que é que os cãezinhos gostam de comer? Eles gostam de ossos! Por isso, cada um deles tem seu próprio osso, de acordo com o seu tamanho. Pois o filho mais novo não pode comer um osso muito grande, e o pai não fica satisfeito apenas com um osso pequenino. Veja o osso de cada cãozinho da família:

\section{Parte B Folha de instrução 2}

Agora, vamos ligar todos os cãezinhos para ir para casa, mas também vamos ligar seus ossos, pois eles estão com fome. Assim, vamos começar novamente pelo filho mais novo, em seguida vamos ligá-lo ao seu osso pequenino. Depois, vamos ligá-lo ao filho do meio, e em seguida ao seu osso, seguido do filho mais velho e também do seu osso e assim por diante. Sem tirar o lápis do papel! Veja o exemplo.

$<$ Exemplo>

<Início da aplicação da parte B do teste>

Páginas de aplicação com os estímulos dos instrumentos podem ser localizados em: Seabra, A. G., \& Dias, N. M. (2012). Avaliação Neuropsicológica Cognitiva: atenção e funções executivas (1st ed.). São Paulo: Memnon.

\section{Quadros Suplementares}

Quadro Suplementar 1. Pontuação-Padrão para a Medida 'Sequências' na Parte A do TT-PE

\begin{tabular}{ccc}
\hline \multirow{2}{*}{$\begin{array}{c}\text { Pontuação } \\
\text { Bruta }\end{array}$} & \multicolumn{2}{c}{ Pontuação Padrão } \\
\cline { 2 - 3 } & 5 anos & 6 anos \\
\hline 1 & 77 & 70 \\
2 & 86 & 80 \\
3 & 94 & 90 \\
4 & 103 & 100 \\
5 & 111 & 110 \\
\hline
\end{tabular}

Quadro Suplementar 2. Pontuação-Padrão para a Medida 'Sequências' na Parte B do TT-PE

\begin{tabular}{ccc}
\hline & \multicolumn{2}{c}{ Pontuação Padrão } \\
\cline { 2 - 3 } Pontuação Bruta & 5anos & 6anos \\
\hline 1 & 87 & 75 \\
2 & 94 & 85 \\
3 & 100 & 96 \\
4 & 107 & 106 \\
5 & 114 & 116 \\
6 & 121 & 126 \\
7 & 127 & 136 \\
8 & 134 & 146 \\
9 & 141 & 156 \\
10 & 148 & 166 \\
\hline
\end{tabular}

Quadro Suplementar 3. Pontuação-Padrão para Acertos na Parte 1 do TAC

\begin{tabular}{ccccccc}
\hline & \multicolumn{2}{c}{ Pontuação Padrão } & & \multirow{2}{*}{$\begin{array}{c}\text { Pontuação } \\
\text { Pontuação Bruta }\end{array}$} & \multicolumn{2}{c}{ Pontuação Padrão } \\
\cline { 7 - 8 } & 5 anos & 6 anos & & Bruta & 5 anos & 6 anos \\
\hline 1 & 56 & 37 & 26 & 94 & 82 \\
2 & 57 & 39 & 27 & 95 & 84 \\
3 & 59 & 41 & 28 & 97 & 86 \\
4 & 60 & 43 & 29 & 98 & 88 \\
5 & 62 & 45 & 30 & 100 & 89 \\
\hline
\end{tabular}




\begin{tabular}{cccccc}
\hline 6 & 63 & 46 & 31 & 101 & 91 \\
7 & 65 & 48 & 32 & 103 & 93 \\
8 & 66 & 50 & 33 & 104 & 95 \\
9 & 68 & 52 & 34 & 106 & 97 \\
10 & 69 & 54 & 35 & 107 & 98 \\
11 & 71 & 55 & 36 & 109 & 100 \\
12 & 72 & 57 & 37 & 110 & 102 \\
13 & 74 & 59 & 38 & 112 & 104 \\
14 & 75 & 61 & 39 & 113 & 106 \\
15 & 77 & 63 & 40 & 115 & 107 \\
16 & 78 & 64 & 41 & 116 & 109 \\
17 & 80 & 66 & 42 & 118 & 111 \\
18 & 81 & 68 & 43 & 119 & 113 \\
19 & 83 & 70 & 44 & 121 & 115 \\
20 & 84 & 71 & 45 & 122 & 116 \\
21 & 86 & 73 & 46 & 124 & 118 \\
22 & 87 & 75 & 47 & 125 & 120 \\
23 & 89 & 77 & 48 & 127 & 122 \\
24 & 90 & 79 & 49 & 128 & 124 \\
25 & 92 & 80 & 50 & 130 & 125 \\
\hline
\end{tabular}

Quadro Suplementar 4. Pontuação-Padrão para Acertos na Parte 2 do TAC

\begin{tabular}{ccc} 
& \multicolumn{2}{c}{ Pontuação Padrão } \\
\cline { 2 - 3 } Pontuação Bruta & 5anos & 6anos \\
\hline 1 & 96 & 89 \\
2 & 108 & 103 \\
3 & 121 & 116 \\
4 & 134 & 129 \\
5 & 146 & 142 \\
6 & 159 & 155 \\
7 & 172 & 168 \\
\hline
\end{tabular}

Quadro Suplementar 5. Pontuação-Padrão para Acertos na Parte 3 do TAC

\begin{tabular}{|c|c|c|c|c|c|}
\hline \multirow[b]{2}{*}{ Pontuação Bruta } & \multicolumn{2}{|c|}{ Pontuação Padrão } & \multirow{2}{*}{$\begin{array}{c}\text { Pontuação } \\
\text { Bruta }\end{array}$} & \multicolumn{2}{|c|}{ Pontuação Padrão } \\
\hline & 5 anos & 6 anos & & 5 anos & 6 anos \\
\hline 1 & 71 & 63 & 27 & 139 & 130 \\
\hline 2 & 73 & 66 & 28 & 142 & 133 \\
\hline 3 & 76 & 68 & 29 & 145 & 135 \\
\hline 4 & 78 & 71 & 30 & 147 & 138 \\
\hline 5 & 81 & 73 & 31 & 150 & 141 \\
\hline 6 & 84 & 76 & 32 & 153 & 143 \\
\hline 7 & 86 & 79 & 33 & 155 & 146 \\
\hline 8 & 89 & 81 & 34 & 158 & 148 \\
\hline 9 & 92 & 84 & 35 & 160 & 151 \\
\hline 10 & 94 & 86 & 36 & 163 & 153 \\
\hline 11 & 97 & 89 & 37 & 166 & 156 \\
\hline 12 & 100 & 92 & 38 & 168 & 159 \\
\hline 13 & 102 & 94 & 39 & 171 & 161 \\
\hline
\end{tabular}




\begin{tabular}{cccccc}
14 & 105 & 97 & 40 & 174 & 164 \\
15 & 108 & 99 & 41 & 176 & 166 \\
16 & 110 & 102 & 42 & 179 & 169 \\
17 & 113 & 104 & 43 & 182 & 172 \\
18 & 115 & 107 & 44 & 184 & 174 \\
19 & 118 & 110 & 45 & 187 & 177 \\
20 & 121 & 112 & 46 & 190 & 179 \\
21 & 123 & 115 & 47 & 192 & 182 \\
22 & 126 & 117 & 48 & 195 & 184 \\
23 & 129 & 120 & 49 & 197 & 187 \\
24 & 131 & 123 & 50 & 200 & 190 \\
25 & 134 & 125 & 51 & 203 & 192 \\
26 & 137 & 128 & 52 & 205 & 195 \\
\hline
\end{tabular}

Quadro Suplementar 6. Pontuação-Padrão para Acertos no TAC (Total)

\begin{tabular}{|c|c|c|c|c|c|}
\hline \multirow{2}{*}{$\begin{array}{l}\text { Pontuação } \\
\text { Bruta }\end{array}$} & \multicolumn{2}{|c|}{ Pontuação Padrão } & \multirow{2}{*}{$\begin{array}{c}\text { Pontuação } \\
\text { Bruta }\end{array}$} & \multicolumn{2}{|c|}{ Pontuação Padrão } \\
\hline & 5 anos & 6 anos & & 5 anos & 6 anos \\
\hline 1 & 54 & 39 & 55 & 112 & 102 \\
\hline 2 & 55 & 40 & 56 & 113 & 104 \\
\hline 3 & 56 & 41 & 57 & 114 & 105 \\
\hline 4 & 57 & 42 & 58 & 115 & 106 \\
\hline 5 & 59 & 44 & 59 & 116 & 107 \\
\hline 6 & 60 & 45 & 60 & 117 & 108 \\
\hline 7 & 61 & 46 & 61 & 118 & 109 \\
\hline 8 & 62 & 47 & 62 & 119 & 111 \\
\hline 9 & 63 & 48 & 63 & 121 & 112 \\
\hline 10 & 64 & 49 & 64 & 122 & 113 \\
\hline 11 & 65 & 51 & 65 & 123 & 114 \\
\hline 12 & 66 & 52 & 66 & 124 & 115 \\
\hline 13 & 67 & 53 & 67 & 125 & 117 \\
\hline 14 & 68 & 54 & 68 & 126 & 118 \\
\hline 15 & 69 & 55 & 69 & 127 & 119 \\
\hline 16 & 70 & 56 & 70 & 128 & 120 \\
\hline 17 & 71 & 58 & 71 & 129 & 121 \\
\hline 18 & 72 & 59 & 72 & 130 & 122 \\
\hline 19 & 73 & 60 & 73 & 131 & 124 \\
\hline 20 & 75 & 61 & 74 & 132 & 125 \\
\hline 21 & 76 & 62 & 75 & 133 & 126 \\
\hline 22 & 77 & 64 & 76 & 134 & 127 \\
\hline 23 & 78 & 65 & 77 & 136 & 128 \\
\hline 24 & 79 & 66 & 78 & 137 & 130 \\
\hline 25 & 80 & 67 & 79 & 138 & 131 \\
\hline 26 & 81 & 68 & 80 & 139 & 132 \\
\hline 27 & 82 & 69 & 81 & 140 & 133 \\
\hline 28 & 83 & 71 & 82 & 141 & 134 \\
\hline 29 & 84 & 72 & 83 & 142 & 135 \\
\hline 30 & 85 & 73 & 84 & 143 & 137 \\
\hline 31 & 86 & 74 & 85 & 144 & 138 \\
\hline
\end{tabular}




\begin{tabular}{llllll}
\hline 32 & 87 & 75 & 86 & 145 & 139 \\
33 & 88 & 76 & 87 & 146 & 140 \\
34 & 90 & 78 & 88 & 147 & 141 \\
35 & 91 & 79 & 89 & 148 & 142 \\
36 & 92 & 80 & 90 & 149 & 144 \\
37 & 93 & 81 & 91 & 150 & 145 \\
38 & 94 & 82 & 92 & 152 & 146 \\
39 & 95 & 84 & 93 & 153 & 147 \\
40 & 96 & 85 & 94 & 154 & 148 \\
41 & 97 & 86 & 95 & 155 & 150 \\
42 & 98 & 87 & 96 & 156 & 151 \\
43 & 99 & 88 & 97 & 157 & 152 \\
44 & 100 & 89 & 98 & 158 & 153 \\
45 & 101 & 91 & 99 & 159 & 154 \\
46 & 102 & 92 & 100 & 160 & 155 \\
47 & 103 & 93 & 101 & 161 & 157 \\
48 & 104 & 94 & 102 & 162 & 158 \\
49 & 106 & 95 & 103 & 163 & 159 \\
50 & 107 & 97 & 104 & 164 & 160 \\
51 & 108 & 98 & 105 & 165 & 161 \\
52 & 109 & 99 & 106 & 167 & 163 \\
53 & 110 & 100 & 107 & 168 & 164 \\
54 & 111 & 101 & & & \\
\hline
\end{tabular}

